Paola María Sánchez Martínez

Revisão Taxonômica da Tribo Dipsadini

(Serpentes: Dipsadidae, Dipsadinae)

São Paulo, Junho de 2016 
Paola María Sánchez Martínez

\title{
Revisão Taxonômica da Tribo Dipsadini \\ (Serpentes: Dipsadidae, Dipsadinae)
}

\author{
Tese apresentada ao Instituto de \\ Biociências da Universidade de São \\ Paulo para obtenção do título de Doutor \\ em Ciências Biológicas \\ Orientador: Dr. Hussam Zaher
}

São Paulo, Junho de 2016 


\section{RESUMO}

A tribo Dipsadini é constituída pelos gêneros Dipsas, Plesiodipsas, Sibon, Sibynomorphus e Tropidodipsas, com 71 espécies válidas que se distribuem desde o México até o norte da Argentina. Os gêneros desta tribo de serpentes Neotropicais apresentam uma extensa variação nos padrões de coloração e na folidose, caracteres que são a base da sistemática deste grupo. Embora tenham sido publicados estudos taxonômicos recentes sobre algumas espécies e grupos de espécies dentro dos gêneros, a taxonomia desses tem se mostrado instável devido ao amplo uso de caracteres morfológicos que apresentam diversas formas intermediárias entre os padrões definidos e, que por sua vez,não são claros. Nesta revisão foram examinados cerca de 1600 exemplares, abrangendo a maioria das espécies da tribo e da sua distribução geográfica. A taxonomia de cinquenta dessas espécies foi avaliada, usando o padrão de coloração, morfologia hemipeniana e caracteres merísticos e morfométricos. As listas sinonímicas de todas as espécies da tribo foram elaboradas, clarificando o status taxonômico atual de cada uma, o que permitiu uma melhor definição taxonômica da tribo. Através desta análise foram reconhecidas 66 espécies válidas, um lectótipo foi designado, seis novas sinonímias foram propostas, cinco espécies foram revalidadas, e quatro espécies foram excluídas da tribo, justificando portanto a necessidade da descrição de dois genêros novos para posicioná-las. De igual forma a descrição de um gênero novo é recomendada para posicionar as espécies de Sibynomorphus de distribuição andina. Já que o status formal de várias das espécies da tribo não é claro, devido ao tratamento indistinto como espécies válidas ou sinônimos, e independentemente dos estudos taxonômicos publicados ao seu respeito, o esclarecimento formal da condição taxonômica dessas espécies é necessário em prol da estabilidade nomenclatural das mesmas. 


\section{ABSTRACT}

The tribe Dipsadini, a group of neotropical snail-eating snakes, includes 71 recognized species, belonging to the genera Dipsas, Plesiodipsas, Sibon, Sibynomorphus and Tropidodipsas, distributed from Mexico to northern Argentina. The genera of this tribe feature an extensive variation of color patterns and scale counts; characters that are the basis of this systematic group. Although taxonomic studies of some species and groups of species within the tribe have been published recently the taxonomy presented has been unstable. This is due to the use of morphological characters that have several intermediate forms between the described species that in turn are not clear. This review included a sample of about 1600 specimens covering the most of the species of the tribe and its geographical distribution. The taxonomic analysis of fifty of these species was carried out using color pattern, hemipenial morphology together with meristic and morphometric characters. The list of synonyms of all species of the tribe was checked to clarify the current taxonomic status of each, which allowed for an improved taxonomic definition of the tribe. In this taxonomic revision 66 valid species were recognized, a lectotype was designated, six species were synonymized, five species have been revalidated, and four species were excluded from the tribe, justifying the need for the two new genus descriptions to place them. Likewise, the description of a new genus is recommended to place the species of Sibynomorphus with Andean distribution. The formal status of several species of the tribe is not clear and these have been treated indistinctly as valid species or synonyms, by different authors. Therefore, the formal clarification of the taxonomic status of these species is necessary prior to any changes being made, in the interests of nomenclatural stability. 


\section{INTRODUÇÃO}

Dipsadidae é uma das maiores famílias de serpentes avançadas (Caenophidia) abarcando mais de 700 espécies, distribuídas exclusivamente nas américas e com a maior diversidade representada por espécies tropicais (Cadle e Greene, 1993). Esta família é definida tanto por caracteres morfológicos, como a presença de espinhos laterais alargados e duas regiões distintamente ornamentadas nos lobos do hemipênis (Zaher, 1999), quanto moleculares (Vidal et al., 2000; Zaher et al. 2009; Vidal et al., 2010; Pyron et al., 2011, 2013). Embora as análises filogenéticas com dados moleculares mais recentes (Zaher et al., 2009; Vidal et al., 2010; Pyron et al., 2011, 2013; Grazziotin et al., 2012) apresentem diferenças no posicionamento de alguns gêneros e na definição de algumas tribos, estas corroboram conjuntamente a condição monofilética da família e dentro desta, o reconhecimento de três linhagens correspondentes a dois grupos biogeográficos (Cadle, 1984a,b,c). Enquanto os gêneros distribuidos na América do Norte (grupo norte-Américano) são alocados por Zaher et al. (2009) na subfamília Carphophiinae e por Vidal et al. (2010) na subfamília Heterodontinae, de forma mais consensual, os gêneros com distribuição predominantemente centro e sul-Américanas, são incluídos respectivamente nas famílias Dipsadinae e Xenodontinae (Cadle, 1984a,b,c, 1988; Zaher et al., 2009; Vidal et al., 2010; Grazziotin et al., 2012).

A subfamília Dipsadinae (sensu Zaher, 1999) é composta por 24 gêneros e aproximadamente 350 espécies de serpentes de hábitos terrícola, arborícola, fossorial ou aquático, (Ferrarezzi, 1994; Fernandes, 1995; Zaher, 1999). Esta subfamília é definida pela presença de hemipênis unilobado ou com bilobação fortemente reduzida, hemipênis unicapitado e bifurcação distal do sulco espermático dentro ou na base do capítulo (Myers, 1974; Cadle, 1984b; Zaher, 1999). Dentre os Dipsadinae são reconhecidas até o momento as tribos Dipsadini (Zaher et al., 2009; Vidal et al., 2010), Imantodini (Daza et al., 2009; Zaher et al., 2009; Vidal et al., 2010; Myers, 2011) e Diaphorolepidini (Pyron et al., 2015). Contudo, vale ressaltar que o número reduzido de gêneros incluídos nas análises moleculares tem impedido a resolução das relações filogenéticas entre os gêneros da subfamília (e.g. Daza et al., 2009 Mulcahy \& Macey, 2009; Grazziotin et al., 2012). 
A tribo Dipsadini (sensu Peters, 1960) foco deste estudo, foi originalmente composta por Dipsas, Sibon e Sibynomorphus, cujas espécies estão distribuídas desde o sul do México até a região norte da Argentina (Peters, 1960). Estes gêneros pertencem a linhagem dos "Goo-eaters", um agrupamento formado por sete gêneros da subfamília Dipsadinae, os quais se alimentam de invertebrados de corpo mole e viscoso, tais como minhocas, lesmas e caracóis (Cadle e Greene, 1993). A tribo Dipsadini (sensu Peters, 1960) é reconhecida desde os trabalhos de Duméril (1853), Günther (1858) e Boulenger (1896) como uma unidade natural, e foi aceita como tal por diversos autores como Underwood (1967), Fernandes (1995) e Wallach (1995). Porém, seu monofiletismo não é corroborado por Dowling e Duellman (1974-1978), Kofron (1985b), Dessauer et al. (1987), Ferrarezzi (1994), Zaher, (1999), Cadle (2007), Harvey et al. (2008), Zaher et al. (2009, 2014), Vidal et al. (2010) e Grazziotin et al. (2012), que incluem gêneros como Adelphicos, Atractus, Cryophis, Geophis, Ninia e Tropidodipsas dentro desse grupo. Assim, apesar de que as relações de parentesco entre Dipsas, Sibon e Sibynomorphus são amplamente aceitas pelos autores anteriormente mencionados, a pouca amostragem e os baixos suportes das filogenias moleculares tem impedido a definição da composição da tribo. Entre os táxons já relacionados com os gêneros Dipsadini, Tropidodipsas é o gênero que aparentemente apresenta a relação de parentesco mais estreita com eles (Cadle, 1984b; Kofron, 1985a; 1987; Dessauer et al. 1987; Zaher, 1999; Cadle, 2007; Harvey et al. 2008; Zaher et al. 2009, 2014), e devido ás evidências que propõem a inclusão desse gênero e de Plesiodipsas em Dipsadini (ver abaixo), aqui considera-se a tribo Dipsadini como definida por Zaher (1999) e Harvey et al. (2008) que agruparam os gêneros Dipsas, Sibon, Sibynomorphus, Plesiodipsas e Tropidodipsas.

As diferenças entre as espécies de Dipsas, Sibon e Sibynomorphus estão concentradas basicamente no padrão de desenho e coloração (Peter, 1960). Esses gêneros se caracterizam pelo seu alto grau de especialização (Peters, 1960; Zaher et al., 20014) que é morfologicamente evidente em característica s como ossos pterigóides curtos e paralelos, corpo lateralmente comprimido, cabeça larga e achatada, olhos proeminentes com pupilas elípticas e ausência de sulcos mentais, exceto em Sibon, para este último caráter (Peters, 1960). Além destas, outras 
característica s morfológicas internas e externas fazem com que os integrantes deste grupo sejam facilmente reconhecidos. Porém, as relações entre estes gêneros e entre as suas espécies permanecem incertas devido principalmente à ampla combinação de caracteres morfológicos com diversas condições "intermediárias" entre os padrões definidos para os gêneros e suas espécies, e também pela carência de revisões taxonômicas e sistemáticas do grupo como um todo (Peters, 1960).

O gênero Dipsas, como é atualmente compreendido, contém 35 táxons nominais (Wallach, 2014) (ver Tabela 1) distribuídos desde o sul do México até a região norte da Argentina (Peters, 1960; Cadle e Myers, 2003; Passos et al. 2004; Cadle, 2005; Harvey, 2008; Harvey \& Embert, 2008). A monografia publicada por Peters (1960) representa o único estudo taxonômico que abrange a totalidade das espécies de Dipsas reconhecidas na sua época. Nessa revisão, Peters (1960) usa principalmente o padrão de coloração e desenho corporal para definir sete grupos de espécies dentro do gênero: articulata, catesbyi, indica, oreas, polylepis, pratti e variegata. Posteriormente, Peters \& Orejas-Miranda (1970) compilam informação sobre 28 espécies de Dipsas, e Fernandes (1995), com base em uma análise cladística, rejeita o monofiletismo do gênero, embora reconheça que a amostra estudada não foi suficiente para testar os agrupamentos propostos por Peters (1960). Estudos mais recentes têm se focado, em espécies ou grupos de espécies dentro do gênero (e.g. Peters, 1970; Fernandes et al., 2002; Cadle e Myers, 2003; Passos et al., 2004; Cadle, 2005; Passos et al., 2005; Fernandes et al., 2010), tentando esclarecer por partes a sua taxonomia. Estudando principalmente as espécies de Dipsas com distribuição na Bolívia, Harvey (2008) e Harvey \& Embert (2008) publicaram uma revisão do gênero na qual redefiniram os grupos propostos por Peters (1960), diganosticaram o gênero com base em caracteres de folidose e morfologia hemipêniana e apresentaram uma chave para as espécies de Dipsas sul-Américanas. Apesar de conter parte das espécies válidas do gênero, estes estudos carecem de uma amostragem abrangente que represente com propriedade a ampla distribuição e a variação morfológica entre as espécies. É especialmente notável a ausência de revisão das populações de Dipsas com distribuição na bacia amazônica e a necessidade de estudos taxonômicos pontuais dentro do gênero, já que decisões nomenclaturais têm sido 
tomadas com base em estudos não taxonômicos, acarretando que algumas das espécies tenham um status taxonômico ambíguo. Como exemplo desta colocação, podemos apontar a catalogação de Wallach (2014), que sem apresentar nenhum comentário taxonômico, considera as seguintes mudanças nomenclaturais: revalidação de $D$. neivai (antes sinônimo junior de $D$. variegata), elevação ao nível de espécie de $D$. indica petersi, e a sinonimização de $D$. bucephala cisticeps e $D$. indica ecuadorensis com $D$. bucephala e $D$. indica, respectivamente.

O gênero Sibon é composto por 16 espécies válidas (Wallach, 2014) (ver Tabela 1) distribuídas principalmente na América Central e norte da América do Sul. A taxonomia do gênero foi revisada por Peters (1960), que com base nos padrões de coloração, definiu três grupos de espécies: annulata, argus e nebulata. Segundo Peters (1960), o gênero se diferença dos demais membros da tribo por apresentar a penúltima escama supralabial mais alta e mais larga que as outras supralabiais. Posteriormente, Kofron (1985a) com base em caracteres de morfologia craniana, hemipeniana e dieta, sinonimizou Tropidodipsas com Sibon, posicionou como incerta sedis as espécies S. carri e S. fischeri, e redefiniu os grupos de espécies em fasciata, sartorii, dimidiata e nebulata, cujas revisões taxonômicas foram publicadas posteriormente por esse mesmo autor (Kofron, 1985b, 1987, 1988 e 1990). As mudanças taxonômicas propostas por Kofron (1985a) foram questionadas por Savage \& McDiarmid (1992) e modificadas por Wallach (1995), que revalidou o gênero Tropidodipsas e o excluiu da tribo Dipsadini (ver detalhes em Wallach, 1995). Porém, os resultados da análise cladística de Fernandes (1995) suportaram a ação de Kofron (1985a) e questionaram os caracteres usados por Wallach (1995). Após estes trabalhos, foram descritas mais sete espécies de Sibon: S. Iamari Solorzano (2001), S. linearis Perez-Higareda et al. (2002), S. manzanaresi McCranie (2007), S. miskitus McCranie (2006), S. perissostichon Köhler et al. (2010), S. merendonensis Rovito, Papenfuss \& Vásquez-Almazán, 2012, e S. noalamina Lotzkat et al. (2012), três das quais foram descritas com base em um único indivíduo.

Sibon nebulatus é a espécie do gênero que apresenta a maior distribuição geográfica, ocorrendo desde o sul do México até o norte do Brasil, incluindo Equador e Trinidad e Tobago (Wallach, 2014). Esta espécie apresenta variaçōes no padrão de desenho e coloração, bem como nas contagens de escamas ventrais e subcaudais, com base nas quais Peters (1960) definiu quatro 
subespécies: nebulatus, leucomelas, hartwegi e popayanensis. As diferenças entre essas subespécies (sensu Peters, 1960) estão basicamente no padrão de manchas dorsais, que podem ser anteriormente largas e bem marcadas (hartwegi) ou pouco definidas e não diferenciadas no resto do corpo (nebulatus), e nas contagens, menos de 175 ventrais e 75 caudais (popayanensis) ou mais de 175 ventrais e 80 subcaudais (leucomelas). No que se refere à distribuição geográfica, a subespécie $S$. $n$. nebulatus é a que apresenta a maior extensão, ocorrendo desde o sul do México até o norte de América do Sul, Trinidad \& Tobago e Equador, chegando até Alagoas no Brasil. As outras três subespécies exibem uma distribuição muito menor, enquanto $S$. $n$. leucomelas ocorre desde o Panamá até o norte do Equador na região cis-andina, as subespécies hartwegi e popayanensis têm uma distribuição restrita à Colômbia, a primeira no curso superior dos rios Ponce e Magdalena e a segunda no curso superior do Rio Cauca. Devido à sobreposição das contagens e à mescla dos padrões de desenho e coloração, a variação descrita no grupo é mal compreendida, e as subespécies descritas por Peters (1960) têm sido ignoradas nos catálogos e bases de dados de serpentes neotropicais (e.g. Wallach et al., 2014; Uetz e Hošek, 2015). Atualmente estas subespécies não são consideradas válidas, embora não haja uma análise de variação posterior a Peters (1960) que suporte tal decisão.

O gênero Sibynomorphus contém 11 espécies válidas (ver Tabela 1), seis das quais ocorrem na Argentina, Brasil, Paraguai, Uruguai e Bolívia, enquanto as demais ocorrem ao longo da costa central do Peru e nas terras baixas das duas vertentes dos Andes no sudoeste do Equador e no norte do Peru (Peters \& Orejas-Miranda, 1970; Franco, 1994; Scrocchi et al., 1993; Cadle, 2007). Ao igual que em Dipsas, o trabalho de Peters (1960) representa o único estudo taxonômico que inclui todas as espécies do gênero Sibynomorphus descritas na época. Peters (1960) reconhece sete espécies de Sibynomorphus e afirma que o gênero é um grupo uniforme que poderia ser constituído apenas de subespécies de S. mikanii, com a exceção de $S$. vagus que realmente poderia manter seu status de espécie. Recentemente, a revisão taxonômica das espécies de Sibynomorphus não andinas foi concluída por Franco (1994) e Scrocchi et al. (1993), e das espécies andinas por Cadle (2007). Além disso, Franco (1994) sugere a revalidação de S. garmani e S. mikanii septentrionalis foi apontada como sinônimo de S. mikanii por Wallach (2014) sem 
apresentar nenhum tipo de evidência taxonômica. Atualmente, estudos taxonômicos destas duas últimas espécies e de $S$. ventrimaculatus, além de uma análise da variação morfológica de S. neuwiedi estão em preparação (F. Franco, 2015 e T. Parnazio, 2015; com. pessoal). No entanto, apesar desses estudos, não há uma análise taxonômica que compare detalhadamente as espécies dos dois grupos geográficos do gênero Sibynomorphus (Cadle, 2007).

A condição parafilética de Dipsas em relação a Sibynomorphus tem sido debatida ao longo dos anos, sem que se tenha alcançado um consenso (e.g. Parker, 1926; Smith e Taylor, 1945; Brongersma, 1958; Kofron, 1990; Fernandes, 1995; Cadle, 2005, 2007; Zaher et al. 2009; Grazziotin, et al. 2012). Segundo Cadle (2005, 2007), Dipsas e Sibynomorphus se diferençam pela presença de dentes no osso pterigóide em Sibynomorphus e a sua ausência em Dipsas, embora Parker (1926) tenha demonstrado que a ausência de dentes pterigoidianos é uma condição presente somente em algumas poucas espécies de Dipsas, já que a maioria possui dentes pterigoidianos na mesma ou em maior quantidade que as espécies de Sibynomorphus. Além disso Cadle $(2005,2007)$ afirma que as diagnoses para Dipsas e Sibynomorphus propostas por Peters (1960) não são suficientes para separar os dois gêneros, e que a evidência do monofiletismo de Sibynomorphus não é conclusiva, porém, este autor não formaliza uma mudança taxonômica para os gêneros, provavelmente considerando que esta seria prematura. De fato, as evidências morfológicas e moleculares que apontam para um possível parafiletismo de Dipsas não são ainda suficientemente abrangentes do ponto de vista amostral para que se possa decidir sobre uma mudança taxonômica coerente para o grupo. Da mesma forma, considera-se que a ampla variação encontrada na morfologia craniana das espécies da tribo Dipsadini (Parker, 1926; Smith \& Taylor, 1945; Brongersma, 1958; Kofron, 1990; Fernandes, 1995; Wallach, 1995) deva ser estudada com mais ênfase, na medida que os problemas taxonômicos sejam resolvidos, e que tal variação deva ser melhor compreendida para que se possa usar essa informação de forma adequada em análises filogenéticas da tribo e da sub-família.

O gênero Tropidodipsas é composto por sete espécies (Wallach, 2014) (ver Tabela 1), que ocorrem desde Nuevo León e Sinaloa, no México, até a Nicarágua, na América Central (Kofron, 
1985). Este gênero se diferença dos demais Dipsadini principalmente pela ausência de um pulmão traqueal extenso (Wallach, 1995) e pelas escamas vertebrais quilhadas e de tamanho similar ás dorsais (Kofron, 1985). Embora Peters (1960) não tenha incluído Tropidodipsas na sua revisão dos Dipsadini, não há dúvidas de que este gênero pertença à tribo Dipsadini, com quem compartilha diversos caracteres derivados exclusivos (Zaher, 1999; Cadle, 2007). Além de apresentar a mesma dieta baseada principalmente em gastrópodes (Cadle, 2007), Tropidodipsas compartilha modificações morfológicas do músculo levator anguli oris que também estão presentes em Sibon (Zaher, 1999), além de caracteres hemipenianos e osteológicos, com base nos quais Kofron (1985a) reconheceu Tropidodipsas e Sibon como congenéricos. Esses caracteres podem ser interpretados como convergências ou como sinapomorfias para o grupo, assim, até não se ter uma análise mais profundada da distribuição e evolução dos mesmos dentro dos Dipsadinae, neste estudo se concorda com Zaher (1999) e Cadle (2007) que incluem o gênero Tropidodipsas dentro da tribo Dipsadini, junto com Dipsas, Sibon e Sibynomorphus. De igual forma, acredita-se que exista uma relação dos gêneros previamente apresentados (Dipsas, Sibon, Sibynomorphus e Tropidodipsas) com o gênero monotípico Plesiodipsas, que é composto por $P$. perijanensis Alemán, 1953, espécie já alocada nos gêneros Tropidodipsas (Alemán, 1953) e Dipsas (Peters, 1970). A distribuição geográfica conhecida de Plesiodipsas é restrita ao nordeste dos Andes colombianos e à Serranía de Perijá na Venezuela. Segundo Harvey et al. (2008), T. perijanensis não pode ser alocada no gênero Dipsas, porque, diferentemente deste, a espécie apresenta um pulmão traqueal curto, uma glândula de Harder grande e 17 escamas dorsais que se reduzem a 15. Do mesmo modo, a espécie não parece pertencer ao gênero Tropidodipsas pela ausência do sulco mental, a penúltima supralabial alargada e escudos mentonianos subretangulares. Dadas essas características, Harvey et al. (2008) criaram o gênero Plesiodipsas para alocar a espécie.

Como exposto acima, a taxonomía das espécies de Sibon, Tropidodipsas e Plesiodipsas têm apresentado várias mudanças nos últimos anos (Kofron, 1985a, 1987; Wallach, 1995; Fernandes, 1995; Bryson \& Lazcano, 2005; Harvey et al. 2008). Apesar de ser evidente que a definição morfológica destes gêneros não é clara, promovendo sua instabilidade, as revisões taxonômicas 
realizadas recentemente (Kofron, 1985a, b; Wallach, 1995; Fernandes, 1995 e Harvey et al. 2008) têm gerado importantes dados para o entendimento do grupo. Porém, alguns destes trabalhos apresentam limitações amostrais que afetam um melhor entendimento e a clara definição dos gêneros (Cadle, 2007).

Grande parte da diversidade apresentada pelos cinco gêneros permanece incompreendida, já que a presença de "formas intermediárias" entre as diagnoses atuais de muitas espécies torna problemática a delimitação dos gêneros em questão, principalmente quando se utiliza a morfologia externa. Apesar da ampla informação disponível nos estudos taxonômicos citados anteriormente, não há uma revisão taxonômica que inclua todas as espécies atuais conhecidas para os cinco gêneros, nem que pretenda avaliar toda a diversidade existente. Assim, os gêneros Dipsas, Plesiodipsas, Sibon, Sibynomorphus e Tropidodipsas requerem uma abordagem conjunta, em um estudo taxonômico amplo e detalhado que inclua o maior número possível de espécies, através da análise de todo o material-tipo disponível e do maior número de exemplares. Esse tipo de estudo é imprescindível para estabelecer e diagnosticar os limites das espécies e para que seja possível a correta atriboição dos seus nomes válidos e a delimitação da distribuição geográfica de cada uma destas. Da mesma forma, uma taxonomia mais apurada das espécies incluídas dentro da tribo proverá informação importante para elaborar hipóteses primárias sobre a definição dos gêneros, as quais deverão ser testadas em análises filogenéticas.

Assim, o intuito deste estudo é realizar uma análise taxonômica que inclua o material disponível dos gêneros Dipsas, Plesiodipsas, Sibon, Sibynomorphus e Tropidodipsas, cujos resultados permitirão definir os limites das espécies e a elaboração das diagnoses dos gêneros com base na morfologia externa e hemipeniana. 


\section{CONCLUSÕES}

Com base na morfologia externa analisada se propõem as seguintes ações taxonômicas, que se justificam no item "comentários" de cada espécie envolvida.

- Sinonimização de Dipsas andiana com D. nicholsi

- Descrição de um gênero novo para Dipsas pakaraima

- Sinonimização de Sibon perissostichon com S. annulatus

- Transferência de Sibon carri para o gênero Geophis

- Sinonimização de Sibon merendonensis com S. miskitus

- Revalidação de Sibon neilli

- Sinonimização de Sibon linearis com S. sanniolus

- Elevação ao nível de espécie de Sibon hartwegi

- Elevação ao nível de espécie de Sibon leucomelas

- Sinonimização de Sibon nebulatus papayanensis com S. hartwegi

- Designação do lectótipo de Sibynomorphus turgidus

- Sinonimização Leptognathus intermedius Steindachner 1903 com S. turgidus

- Descrição de um gênero novo para as espécies andinas de Sibynomorphus

- Revalidação de Tropidodipsas guerreroensis Taylor,1939

- Descrição de um gênero novo para Tropidodipsas fischeri e T. kidderi 


\section{REFERÊNCIAS BIBLIOGRÁFICAS}

ACOSTA-CHAVES, V., GARITA, C., CONEJO-BARBOZA, K., RAMIREZ-FERNANDEZ, J.D., NARANJO, B. e JIMENEZ, A. 2014. Geographic Distribution: Sibon anthracops (Cope's snail sucker). Herpetological Review, 45 (3): 467.

ALEIXO, A. 2007. Conceitos de espécie e o eterno conflito entre continuidade e operacionalidade: uma proposta de normatização de critérios para o reconhecimento de espécies pelo Comitê Brasileiro de Registros Ornitológicos. Revista Brasileira de Ornitologia, 15: 229-242.

ALEMÁN G. C. 1953. Apuntes sobre reptiles y anfibios de la región Baruta-El Hatillo. Memoria de la Sociedad de Ciencias Naturales La Salle, 12 (31): 11-30 [1952].

ALVES, F.Q., ARGOLO, A.J., 1998. Geographic distribution. Dipsas indica petersi. Herpetological Review, 29 (3): 176

AMARAL, A. do. 1929. Estudos sobre ophídios neotrópicos. XVIII. Lista remissiva dos ophidios da região neotropica. Memórias do Instituto Butantan, São Paulo (1929) 4, 129-271.

BOIE, F. 1827. Bemerkungen über Merrem's Versuch eines Systems der Amphibien. Marburg, 1820. 1te Lieferung, Ophidier. Isis von Oken, Jena 20(6), 508-566.

BOULENGER, G. A. 1896. Catalogue of the Snakes in the British Museum (Natural History). Vol. III. British Museum (Natural History), London.

BOULENGER, G.A. 1897. Description of a new snake from the Andes of Colombia. Annals and Magazine of Natural History, London (Series 6) 20(120), 523.

BRONGERSMA, L. D. 1958. Some Features of the Dipsadinae and Pareinae (Serpentes, Colubridae). Proceedings, Series Koninkl Nederl. Akademie Van Wetenschappen, (C) 61: 7-12.

CADLE, J. E. 1984 a. Molecular systematics of Neotropical xenodontine snakes. I. South American xenodontines. Herpetologica, 40:8-20.

CADLE, J. E. 1984 b. Molecular systematics of Neotropical xenodontine snakes. II. Central American xenodontines. Herpetologica, 40:21-30.

CADLE, J. E. 1984 c. Molecular systematics of Neotropical xenodontine snakes. III. Overview of xenodontine phylogeny and the history of New World snakes. Copeia, 1984:641-652.

CADLE, J. E. 1988. Phylogenetic relationships among advanced snakes: a molecular perspective. University of California Publications in Zoology, 119:1-70. 
CADLE, J. E. 2005. Systematics of snakes of the Dipsas oreas complex (Colubridae: Dipsadinae) in western Ecuador and Peru, with revalidation of D. elegans (Boulenger) and D. ellipsifera (Boulenger). Bulletin Museum of Comparative Zoology, 158(3): 67-136.

CADLE, J. E. 2007. The snake genus Sibynomorphus (Colubridae: Dipsadinae: Dipsadini) in Peru and Ecuador, with comments on the systematics of Dipsadini. Bulletin Museum of Comparative Zoology, 158(5): 183-284.

CADLE, J. E. e GREENE, W. 1993. Phylogenetic patterns, Biogeography, and the ecological structure of Neotropical snake assemblages. Em: Ricklefs, R. E. e Schluter, D. (eds.). Species Diversity in Ecological Communities: Historical and Geographical Perspectives. Chicago: Chicago Press. p. 281-293.

CADLE, J. E. e MYERS, C. W. 2003. Systematics of snakes referred to Dipsas variegata in Panama and Western South America, with revalidation of two species and notes on defensive behaviors in the Dipsadini (Colubridae). American Museum Novitates, 3409:1-47.

CARRILLO DE ESPINOZA, N. 1974 Sibynomorphus williamsi nov. sp. (Serpentes, Colubridae). Publicaciones del Museo de Historia Natural "Javier Prado", (Zoología), Lima (24A), 1-16, 8 figs.

CICCHI, P.J.P., SENA, M.A. , PECCININI-SEALE, D.M. e DUARTE, M.R. 2007. Snakes from coastal islands of State of São Paulo, Southeastern Brazil. Biota Neotropica, 17:227-240.

COPE, E.D. 1868. An examination of the Reptilia and Batrachia obtained by the Orton Expedition to Ecuador and the Upper Amazon, with notes on other species. Proceedings of the Academy of Natural Sciences of Philadelphia, 20(2), 96-119.

COPE, E.D. 1874; Description of some species of reptiles obtained by Dr. John F. Bransford, Assistant Surgeon United States Navy, while attached to the Nicaraguan Surveying Expedition in 1873.Proceedings of the Academy of Natural Sciences of Philadelphia, 26, 64-72 [65].

COPE, E.D. 1875. On the Batrachia and Reptilia of Costa Rica. With notes on the herpetology and ichthyology of Nicaragua and Peru. Journal of the Academy of Natural Sciences of Philadelphia, (1876) (Series 2) 8(2), 93-154, pls. 23-28.

COPE, E.D. 1879. Eleventh contribution to the herpetology of tropical America. Proceedings of the American Philosophical Society, (1878-1880) 18(104), 261-277.

COPE, E.D. 1887. Synopsis of the Batrachia and Reptilia obtained by H.H. Smith, in the province of Mato Grosso, Brazil. Proceedings of the American Philosophical Society 24, (125):44-60.

COSTA, H.C. e BÉRNILS, R.S. 2014. Répteis brasileiros: Lista de espécies. Herpetologia Brasileira, 3 (3), 7484. 
CRACRAFT, J., 1983. Species concept and speciation analysis. In: Johnston, R. F. (ed.), Current Ornithology, Volume 1. Plenum Press, New York.

CUNHA, O.R. da, do NASCIMENTO, F.P. e HOGE, A.R. 1980. Ofídios da Amazônia. XII. Uma subspécie de Sibynomorphus mikani do noroeste do Maranhão (Ophidia, Colubridae, Dipsadinae). Boletim do Museu Paraense Emílio Goeldi, (Zoologia), Belém (103), 1-15.

DAZA, J. M., SMITH, E. N., PAEZ, V. P. e PARKINSON, C. L. 2009. Complex evolution in the Neotropics: the origin and diversification of the widespread genus Leptodeira (Serpentes: Colubridae). Molecular Phylogenetics and Evolution, 53(3):653-667.

DESSAUER, H. C., CADLE, J. E. e LAWSON, R. 1987. Patterns of snake evolution suggested by their proteins. Fieldiana, Zoology, ser. 2(34):1-34.

DOWLING, H. G. 1951. A proposed standard system of counting ventrals in snakes. British Journal of Herpetology, 1:97-99.

DOWLING, H. G. e DUELLMAN, W. E. 1974-1978. Systematic Herpetology: a Synopsis of Families and Higher Categories. New York: Hiss Publication.

DOWNS, F.L. 1967. Intrageneric relationships among colubrid snakes of the genus Geophis Wagler. Miscellaneous Publications, Museum of Zoology, University of Michigan, (131), 1-193.

DUELLMAN, W.E. (1979) The South American herpetofauna: its origin, evolution, and dispersal. Monographs, University of Kansas Museum of Natural History (7), 1-485, 172 figs

DUNN, E.R. 1942. New or noteworthy snakes from Panama. Notulae Naturae, Philadelphia, (108), 1-8.

DUMÉRIL, A. M. C. 1853. Prodrome de la classification des reptiles ophidiens. Firmin Didot Frères, Paris.

DUMÉRIL, A.M.C., BIBRON, G. \& DUMÉRIL, A.H.A. 1854. Erpétologie générale ou histoire naturelle complète des reptiles. Tome septième - Premiere partie. Comprenant l'histoire des serpents non venimeux. Librairie Encyclopédique de Roret, Paris, vii + 780 pp.

DWYER, Q. 2015. Geographic Distribution. Sibon anthracops (Ringed Snaileater). Herpetological Review, 46: 63.

FERNANDES, R. 1995. Phylogeny of the Dipsadine snakes. Tese (Doutorado) University of Texas at Arlington. Arlington, 1995.

FERNANDES, R., PORTO, M. \& CARAMASCHI, U. 1998. The taxonomic status of Heterorhachis poecilolepis Amaral, 1923. Journal of Herpetology, 32(1), 139-141. 
FERNANDEZ, R., FERNANDES, D. S. e PASSOS, P. 2002. Leptognathus latifasciatus Boulenger, 1913, a Junior synonym of Dipsas polylepis (Boulenger, 1912) (Serpentes, Colubridae). Boletim do Museu Nacional, Nova Série, Zoologia, 493, 1-7.

FERNANDES, D. S, MARQUES, O. A. e ARGÔLO, A. J. S. 2010. A new species of Dipsas Laurenti from the Atlantic Forest of Brasil (Serpentes: Dipsadidae). Zootaxa, 2691: 57-66.

FERRAREZZI, H. 1994. Uma sinopse dos gêneros e classificação das serpentes (Squamata) II. Família Colubridae. In: Nascimento, L. B.; Bernardes, A. T. e Cotta, G. A. (eds.). Herpetologia no Brasil. Belo Horizonte, PUC-MG; Fundação Biodiversitas e Fundação Ezequiel Dias. v. 1, p. 81-91.

FJELDSÄ, J., ÁLVAREZ, M.D., LAZCANO, J.M., e LEÓN, B. 2005. Illicit crops and armed conflict as constraints on biodiversity conservation in the Andes region. AmBio, 34:205-211.

FRANCO, F. L. 1994. O gênero Sibynomorphus Fitzinger,1843, no Brasil (Colubridae; Xenodontinae; Dipsadini). Dissertação (Mestrado), Pontificia Universidade Católica do Rio Grande do Sul, Rio Grande do Sul, 1994.

FREITAS, M. A. de, ALMEIDA, A. B., ALMEIDA, M., SALGADO, T., MOURA, G.J., 2014. Rediscovery and first record of Sibynomorphus mikanii septentrionalis (Cunha, Nascimento e Hoge, 1980), (Squamata; Serpentes) for the state of Pará. Check List, 10 (5): 1246-1248.

GOOGLE EARTH WEBSITE. 2009. http://earth.google.com/

GRAZZIOTIN, F. G., ZAHER, H., MURPHY, R., SCROCCHI, G., BENAVIDES, M. A., ZHANG, Y. e BONATTO, S. L. 2012. Molecular phylogeny of the New World Dipsadidae (Serpentes: Colubroidea): a reappraisal. Cladistics, 1: 1-23.

GÜNTHER, A. C. L. G. 1858. Catalogue of Colubrine Snakes in the Collection of the British Museum. British Museum (Natural History), London).

GÜNTHER, A.C.L.G. 1872 Seventh account of new species of snakes in the collection of the British Museum. Annals and Magazine of Natural History, London (Series 4) 9(49), 13-37.

HARVEY, M. B. 2008. New and poorly known Dipsas (Serpentes: Colubridae) from northern South America. Herpetologica, 64, 422-451.

HARVEY, M. B. e EMBERT, D. 2008. Review of Bolivian Dipsas (Serpentes: Colubridae), with comments on other South American species. Herpetological Monographs, 22, 54-105. 
HARVEY, M. D., RIVAS, G., CAICEDO, J. R., RUEDA-ALMONACID, J. 2008. Systematics of the enigmatic Dipsadine snake Tropidodipsas perijanensis Alemán (Serpents: Colubridae) and review of morphological characters of Dipsadini. Herpetological Monographs, 22: 106-132.

HIDALGO, H. N. 1979. Range extension of the snake Sibon anthracops (Cope) in El Salvador. Herpetological Review, 10 (3): 103.

HOFMANN, E.P. 2016. Geographic Distribution: Sibon dimidiatus (Slender Snail Sucker). Herpetological Review, 47 (1): 84.

HOGE, A.R. 1950. Notas erpétologicas. 7. Fauna erpetológica da Ilha da Queimada Grande. Memórias do Instituto Butantan, São Paulo (1949) 22, 151-171.

HOGE, A.R. 1969. Notes on the holotype of Dipsas indica cisticeps (Boettger) (Serpentes-Dipsadinae). Memórias do Instituto Butantan, 34:87-88.

HOGE, A.R. e ROMANO-HOGE, S.A.R.W.L. 1975. A new subspecies of Dipsas indica from Brazil [Serpentes, Colubridae, Dipsadinae]. Memórias do Instituto Butantan, 39: 51-60.

IHERING, R. von, 1911. As cobras do Brazil. Revista do Museu Paulista, São Paulo, 8, 273-379.

IUCN. 1982. International Union for Conservation of Nature and Natural Resources. Directory of Neotropical Protected areas. Tycooly International, Dublin, 436 pp.

JARAMILLO A., IBAÑEZ, R.D., JARAMILLO, F.E., e SOLIS, F.A. 2008. Geographic distribution: Sibon lamari. Herpetological Review 39 (3): 373-374.

JAN, G. 1863. Elenco sistematico degli ofidi descritti e disegnati per l'Iconografia générale. A. Lombardi, Milano, vii + 143 pp.

JAN, G. e SORDELLI, F. 1860. Iconographie general des ophidiens. Milan and Paris. Pp. 100 (in 2 vols.), Atlas 1881 (3 vols.).

KOFRON, C.P. 1982a The identities of some dipsadine snakes: Dipsas elegans, D. ellipsifera and Leptognathus andrei. Copeia 1982(1), 46-51

KOFRON, C.P. 1982b. A review of Mexican snail-eating snakes, Dipsas brevifacies and Dipsas gaigeae. Journal of Herpetology, 16(3), 270-286.

KOFRON, C. P. 1985a. Systematics of the Neotropical Gastropod-eating Snake Genera, Tropidodipsas and Sibon. Journal of Herpetology, 19(1):84-92. 
KOFRON, C. P. 1985b. Review of the Central American Colubrid snakes, Sibon fischeri and S. carri. Copeia, 1985: 164-174.

KOFRON, C. P. 1987. Systematics of Neotropical gastropod-eating snakes: the fasciata group of the genus Sibon. Journal of Herpetology, 21 (3): 210-225.

KOFRON, C. P. 1988. Systematics of Neotropical gastropod-eating snakes: the sartorii group of the genus Sibon. Amphibia-Reptilia, 9:145-168

KOFRON, C. P. 1990. Systematics of neotropical gastropod eating snakes: the dimidiata group of the genus Sibon, with comments on the nebulata group. Amphibia-Reptilia, 11:207-223.

KÖHLER, G., SEIPP, R. 1998. Geographic distribution. Sibon annulatus. Herpetological Review, 29 (4): 249.

KÖHLER, G., LOTZKAT, S. e HERTZ, A. 2010. A new species of Sibon (Squamata: Colubridae) from western Panama. Herpetologica, 66 (1): 80-85.

KORNACKER, P., La MARCA, E., e RIVAS, G. 2010. Plesiodipsas perijanensis. IUCN Red List of Threatened Species. Versión 2015.4. Base de datos accesible en: http://www.iucnredlist.org/. Acceso el 29 de noviembre 2015.

LEMA, T. de. 2002. Os répteis do Rio Grande do Sul: atuais e fósseis, biogeografia, ofidismo. Porto Alegre: EDIPUCRS. Não paginado.

LEMOS-ESPINAL, J.A. e SMITH, H.M. 2007. Amphibians and reptiles of the state of Chihuahua, Mexico. Universidad Nacional Autónoma de México, Mexico City, xii + 613 pp., 129 figs., 249 fotos.

LEWIS, T.R., GRIFFIN, R.K. GRANT, P.B.C., FIGUEROA, A., RAY, J.M., GRAHAM, K.E. e DAVID, G. 2013. Morphology and ecology of Sibon snakes (Squamata: Dipsadidae) from two Neotropical forests in Mesoamerica. Phyllomedusa, 12 (1): 47-55.

LINER, E.A. e WILSON, L.D. 1970. Changes in the name and generic status of the Mexican snake Chersodromus annulatus Zweifel (Colubridae). Copeia, 1970(4), 786-788.

LOTZKAT, S., HERTZ, A., KÖHLER, G. 2012. A new species of Sibon (Squamata: Colubroidea: Dipsadidae) from the Cordillera Central of western Panama, with comments on other species of the genus in the area Zootaxa, 3485: 26-40.

MALNATE, 1971, A Catalog of Primary Types in the Herpetological Collections of the Academy of Natural Sciences, Philadelphia (ANSP). Proc. Acad. Nat. Sci. Philadelphia, 123:345-375[363].

MANZANI, P. R. e ABE, A. S. 1988. Sobre dois novos métodos de preparo do hemipênis de serpentes. Memórias do Instituto Butantan, 50(1): 15-20. 
MAYR, E., 1942. Systematics and the origin of species. Columbia University Press, New York.

MCCRANIE, J.R. 2006. New species of Sibon (Squamata: Colubridae) from Northeastern Honduras. Journal of Herpetology, 40 (1): 16-21

MCCRANIE, J.R. 2007. A second new species of Sibon (Squamata: Colubridae) from La Mosquitia, Northeastern Honduras. Herpetologica, 63 (2): 213-218.

MacCULLOCH, R.D. e LATHROP, A. 2004. A new species of Dipsas (Squamata: Colubridae) from Guyana. Revista de Biologia Tropical, 52(1), 239-247

McDIARMID, R.W., CAMPBELL, J.A. e TOURÉ, T.A. 1999. Snake species of the world. Vol. 1. Herpetologists' League, $511 \mathrm{pp}$.

MORENO-ARIAS, R. 2010. Geographic distribution: Sibon annulatus. Herpetological Review, 41 (3): 382.

MULCAHY, D. G. e MACEY, J. R. 2009. Vicariance and dispersal form a ring distribution in night snakes around the Gulf of California. Molecular Phylogenetics and Evolution, 53: 537-546.

MURPHY, J. e RUTHERFORD, M.G., 2014. The snail-eating snake Dipsas variegata (Duméril, Bibron and Duméril) on Trinidad, and its relationship to the microcephalic Dipsas trinitatis Parker (Squamata, Dipsadidae) Herpetology Notes, volume 7: 757-760.

MYERS, C. W. 1974. The systematic of Rhadinaea (Colubridae), a genus of New World snakes. Bulletin of the American Museum of Natural History, 153:1-262.

MYERS, C. W. 2011. A New Genus and New Tribe for Enicognathus melanauchen Jan, 1863, a Neglected South American Snake (Colubridae: Xenodontinae), with Taxonomic Notes on Some Dipsadinae. American Museum Novitates, 3715:1-33.

MYERS, C. W. e CAMPBELL, J. A. 1981. A new genus and species of colubrid snake from the Sierra Madre del Sur of Guerrero, Mexico. American Museum Novitates, 2708: 1-20.

MYERS, C. W. e TRUEB, L. 1967. The hemipenis of an anomalepidid snake. Publisher Herpetologica, 23 (3): 235-238.

MÜLLER, F. 1878. Mittheilungen aus der herpetologischen Sammlung des Basler Museums. I. Ueber einige seltene und neue Reptilien aus Guatémala. Verhandlungen der Naturforschenden Gesellschaft in Basel (1877) 6(3), 389-411.

MÜLLER, F. 1887. Fünfter Nachtrag zum Katalog der herpetologischen Sammlung des Basler Museums. Verhandlungen der Naturforschenden Gesellschaft in Basel (1887-1890) 8(2), 249-296. 
OlSON, D. M., Dinerstein, E. C., Wikramanayake, E. D., BURgeSS, N. D., POWELL, G. V. N., UNDERWOOD, E. C., D’AMICO, J. A., ITOUA, I., STRAND, H. E., MORRISON, J. C., LOUCKS, C. J., ALLNUTT, T. F., RICKETTS, T. H., KURA, Y., LAMOREUX, J. F., WETTENGEL, W. W., HEDAO, P. e KASSEM, K.R. 2001. Terrestrial Ecoregions of the World: A New Map of Life on Earth. BioScience, (51) 1:933-9381.

PARKER, H. W. 1926. Description of a new snake from Trinidad. Annals and Magazine of Natural History, series $9,18: 205-207$.

PARKER, H. W. 1934. Reptiles and amphibians from southern Ecuador. The Annals and Magazine of Natural History 14:264-273.

PASSOS, P., FERNANDES, D. S. e CARAMASCHI, U. 2004. The taxonomic status of Leptognathus incertus Jan, 1863, with revalidation of Dipsas alternans (Fischer, 1885) (Serpentes: Colubridae: Dipsadinae). Amphibia-Reptilia, 25:381-393.

PASSOS, P., FERNANDES, R. e PORTO, M. 2005. Geographical variation and taxonomy of the snail-eating snake Dipsas albifrons (Sauvage, 1884), with comments on the systematic status of Dipsas albifrons cavalheiroi Hoge, 1950 (Serpentes: Colubridae: Dipsadinae). Zootaxa, 1013, 19-34.

PERCEQUILLO, R. A. 2003. Sistemática de Oryzomys Baird, 1858: definição dos grupos de espécie e revisão taxonômica do grupo albigularis (Rodentia, Sigmodontinae). Tese (Doutorado), Universidade de São Paulo. São Paulo, 2003.

PÉREZ-HIGAREDA, G. LÓPEZ-LUNA, M. A. e SMITH, H. M. 2002. A new snake related to Sibon sanniola (Serpentes: Dipsadidae) from Los Tuxtlas, Veracruz, Mexico. Bulletin Maryland Herpetological Society, 38(2):62-65.

PESANTES, O. S. 1994. A method for preparing the hemipenis of preserved snakes. Journal of Herpetology, 28: 93-95.

PETERS, J. 1957 A New Snake of the Genus Sibon from Ecuador. Copeia, No. 2: 109-11.

PETERS, J. A. 1960. The snakes of the subfamily Dipsadinae. Miscellaneus Publications Museum of Zoology, University of Michigan 114:1-224.

PETERS, J. A. 1970. Generic position of the South American snake Tropidodipsas perijanensis, Copeia: 394395.

PETERS, J. A. e OREJAS-MIRANDA, B. 1970. Catalogue of the Neotropical Squamata. Part I: Snakes. Smithsonian Institution Press, Washington.

PORTO, M. e FERNANDES, R. 1996. Variation and natural history of the snail-eating snake Dipsas neivai (Colubridae: Xenodontinae). Journal of Herpetology, 30 (2): 269-271. 
PRADO, A. 1941. Notas ofiológicas. 6. Uma nova espécie de colubrideo áglifo de Colômbia. Memórias do Instituto Butantan, São Paulo (1940) 14, 13-15.

PYRON, R. A., BURBRINK, F. T., COLLI, G. R., MONTES DE OCA, A. N., VITT, L. J., KUCZYNSKI, C. A. e WIENS, J. J. 2011. The phylogeny of advanced snakes (Colubroidea), with discovery of a new subfamily and comparison of support methods for likelihood trees. Molecular Phylogenetics and Evolution, 58(2): 329-342.

PYRON, R.A., BURBRINK, F.T., e J.J. WIENS. 2013. A phylogeny and updated classification of Squamata, including 4161 species of lizards and snakes. BMC Evolutionary Biology, 13: 93.

PYRON, R.A., GUAYASAMIN, J.M., PEÑAFIEL, N., BUSTAMANTE, L., e A. ARTEAGA. 2015. Systematics of Nothopsini (Serpentes: Dipsadidae), with a new species of Synophis from the Pacific Andean slopes of southwestern Ecuador. Zookeys, 541: 109-147.

RANGEL-CH, J. O. 2007. La alta montaña de Perijá: consideraciones finales. Pp. 417-436. En: J. O. RangelCh (Editor). Colombia Diversidad Biótica V: La Alta Montaña de la Serranía de Perijá. Instituto de Ciencias Naturales, Universidad Nacional de Colombia, Bogotá, Colombia.

RANGEL-CH, J. O., AGUILAR-P.M., e LOWY-C. 1995. Parque Nacional Natural Sierra de la Macarena. Pp.112-120.Em J. O. Rangel-Ch. (editor), Colombia: Diversidad Biotica, Vol. 1. Universidad Nacional de Colombia, Bogotá.

RYAN, M.L., LATELLA, I.M., WILLINK, B., GARCIA-RODRIGEUZ, A. e GILMAN, C.A. 2015. Notes on the breeding habits and new distribution records of seven species of snakes from southwest Costa Rica. Herpetology Notes, 8: 669-671.

RAY, J.M., MONTGOMERY, C.E., MAHON, H.K., SAVITZKY, A.H., e LIPS, K.R. 2012. Goo-Eaters: Diets of the Neotropical Snakes Dipsas and Sibon in Central Panama. Copeia, 2012 (2): 197-202.

REYNOLDS, R.P. e FOSTER, M.S. 1992. Four new species of frog and one new species of snake from the Chapare region of Bolivia, with notes on other species. Herpetological Monographs, 6:83-104.

ROSSMAN, D. A., KIZIRIAN, D. A. 1993. Variation in the peruvian dipsadine snakes Sibynomorphus oneilli and S. vagus. Journal of Herpetology, 27 (1): 87-90.

ROSSMAN, D.A. e THOMAS, R. 1979. A new dipsadine snake of the genus Sibynomorphus from Peru. Occasional Papers, Museum of Zoology, Louisiana State University 54:1-6. .

ROVITO, S.M., Papenfuss, T.J., e VÁSQUEZ-ALMAZÁN, C.R. 2012. A new species of Sibon (Squamata: Colubridae) from the mountains of eastern Guatemala. Zootaxa, 3266, 62-68. 
ROVITO, S.M., DEVITT, T. J., DEVITT, S. C. 2015. First survey of the amphibians and reptiles of the Nectandra Cloud Forest Reserve, Alajuela, Costa Rica. Check List, 11(2): 1570.

SÁNCHEZ-MARTíNEZ. P.M. 2007. Anatomia Comparada da tribo Nothopsini (Serpentes, Dipsadidae). Dissertação (Mestrado) - Departamento de Zoologia, Instituto de Biociências, Universidade de São Paulo. São Paulo, 2007.

SAVAGE, J. M. 2002. The Amphibians and Reptiles of Costa Rica. Chicago: University of Chicago Press, 934 pp.

SAVAGE, J. M., e McDiarmid, R. W. 1992. Rediscovery of the Central American colubrid snake, Sibon argus, with comments on related species from the region. Copeia, 1992(2): 421-432.

SCHELEGEL, H. 1837. Essai sur la physionomie des serpents. I. Partie générale. II. Partie descriptive. Atlas, 21 planches et 3 cartes. Arnz Arnz e Comp., Leiden, xxviii $+251+x v i ., 606$ pp.

SCOTT. N.J. 1967. The colubrid snake, Tropidodipsas annulifera, with reference to the status of Geatractus, Exelencophis, Chersodromus annulatus, and Tropidodipsas malacodryas. Copeia, 1967 (2): 280-287.

SCROCCHI, G.; PORTO, M. e REY, L. 1993. Descripción de una espécie nueva y situación del género Sibynomorphus (Serpentes: Colubridae) en la Argentina. Revista Brasileira de Biologia, 53 (2): 197208.

SEBA, A. 1734 Locupletissimi rerum naturalium thesauri accurata descriptio, et iconibus artificiosissimis expressio, per universam physices historiam, Amsterdam. Val. I. P. 1734.

SENTZEN, U. J. 1796. Ophiologische Fragmente No. 6. Beschreibung des Coluber Catesbeji. Meyer's Zool. Arch., 2: 66-74.

SHEEHY, C. M. 2012. Phylogenetic Relationships and Feeding Behavior of Neotropical Snail-eating Snakes (Dipsadinae, Dipsadini). Tese (Doutorado), University of Texas at Arlington. Arlington, 2012.

SIMPSON, G. G., 1961. Principles of animal taxonomy. Columbia University Press, New York.

SMITH, H.M., 1943. Summary of the collections of snakes and crocodilians made in Mexico under the Walter Rathbone Bacon Traveling Scholarship. Proceedings of the United States National Museum, 93:393-504.

SMITH, H. M. e TAYLOR, E. H. 1945. An annotated checklist and key to the snakes of Mexico. Bulletin of the United States National Museum, 187: iv + 1-239.

SOLÓRZANO, A. 2001. Una nueva espécie de serpiente del genero Sibon (Serpentes: Colubridae) de la vertiente del Caribe de Costa Rica. Revista de Biologia Tropical, 49(3-4): 1111-1120 
SOLÓRZANO, A. 2004. Serpientes de Costa Rica: distribución, taxonomía e historía natural. Snakes of Costa Rica: distribution, taxonomy, and natural history. Instituto Nacional de Biodiversidad (NBio), Santo Domingo de Heredía, 792 pp.

SOLÓRZANO, A. 2006. Snakes of the Osa Peninsula [Costa Rica]. Reptilia (GB) (48): 30-34.

SOLIS, M.E. e MARIO, J. 2015. New locality and elevational record for the snake Sibon anthracops (Cope, 1868) in Honduras. Mesoamerican Herpetology, 2 (2): 218-219.

STARK, T., LAURIJSSENS, C., E. WETERINGS. W. 2014. Distributional and natural history notes on five species of amphibians and reptiles from Isla Ometepe, Nicaragua. Mesoamerican Herpetology, 1 (2): 308.

STUART, L.C. 1963. A checklist of the herpetofauna of Guatemala. Miscellaneous Publications, Museum of Zoology, University of Michigan, 122:1-152.

TAYLOR, E.H. 1940. Some Mexican serpents. Bulletin of the University of Kansas, 26 (14): 445-87.

TAYLOR, E.H. 1954. Further studies on the serpents of Costa Rica. Bulletin of the University of Kansas, 36 : 673-800.

TIEDEMANN, F., HÄUPL, M. e GRILLITSCH, H. 1994. Katalogen der Typen der Herpetologischen Sammlung nach dem Stand vom 1. Naturhistorischen Museums in Wien. Jännerd.

UETZ, P. e HOŠEK, J., (eds.), 2016. The Reptile Database, http://www.reptile-database.org

UNDERWOOD, G. L. 1967. A Contribution to the Classification of Snakes. British Museum of Natural History, London: British Museum (Natural History). x, 179 p.

VANZOLINI, P.E. 1953. On the Type Locality of Some Brazilian Reptiles and Amphibians Collected by H. H. Smith and Described by E. D. Cope. Copeia, 2: 124-125.

VIDAL, N., KINDL, S., WONG, A. e HEDGES, B. 2000. Phylogenetic Relationships of Xenodontine Snakes Inferred from $12 S$ and $16 S$ Ribosomal RNA Sequences. Molecular Phylogenetics and Evolution, 14(3) 389-402.

VIDAL, N., DEWYNTER, M. e GOWER, D. J. 2010. Dissecting the major American snake radiation: A molecular phylogeny of the Dipsadidae Bonaparte (Serpentes, Caenophidia). Comptes Rendus Biologies, 333: 48-55.

WALLACH, V. 1995. Revalidation of the genus Tropidodipsas Günther, with notes on the Dipsadini and Nothopsini (Serpentes: Colubridae). Journal of Herpetology, 9(3):476-481. 
WALLACH, V., WILLIAMS, K.L., BOUNDY, J. 2014. Snakes of the World: A Catalogue of Living and Extinct Species. CRC Press, Boca Rattan, FL. 1237 pp.

WILSON, L.D., e MEYER, J.R. 1985. The snakes of Honduras, 2d ed. Milwaukee Pub. Mus.1-150. (JMS) DEPARTMENT OF BIOLOGY.

WIENS, J. J. e SERVEDIO, M. R. 2000. Species delimitation in systematics: inferring diagnostic differences between species. Proceedings of the Royal Society of London, 267: 631-636.

ZAHER, H. 1999. Hemipenial morphology of the South American xenodontine snakes, with a proposal for a monophyletic Xenodontinae and a reappraisal of Colubrid hemipenes. Bulletin of the American Museum of Natural History, 240: 1-168.

ZAHER, H. e PRUDENTE, A. L. 1999. Intraspecific variation of the hemipenis of Siphlophis and Tripanurgos (Serpentes, Xenodontinae). Journal of Herpetology, 33(4): 698-702.

ZAHER, H., GRAZZIOTIN, F. G., CADLE, J.E., MURPHY, R. W., MOURA-LEITE, J.C., BONATTO, S. L. 2009. Molecular phylogeny of advanced snakes (Serpentes, Caenophidia) with an emphasis on South American xenodontines: a revised classification and descriptions of new taxa. Papéis Avulsos de Zoologia, 49: 115-153.

ZAHER, H., OLIVEIRA, L. GRAZZIOTIN, F.G., CAMPAGNER, M., JARED, C., ANTONIAZZI, M., e PRUDENTE, A. 2014 Consuming viscous prey: a novel protein-secreting delivery system in neotropical snail-eating snakes. BMC Evolutionary Biology, 14:58. 